\title{
Surgical fixation of rib fractures decreases intensive care length of stay in flail chest patients
}

\author{
Xiangzhi Xiao" ${ }^{1 \#}$, Shengchao Zhang ${ }^{1 \#}$, Juhua Yang ${ }^{1}$, Jian Wang ${ }^{2}$, Zhilong Zhang ${ }^{2}$, Hao Chen ${ }^{1,2}$ \\ ${ }^{1}$ Department of Thoracic Surgery, Qingpu Branch of Zhongshan Hospital, Fudan University, Shanghai 201700, China; ${ }^{2}$ Department of Thoracic \\ Surgery, Xuhui Branch of Zhongshan Hospital, Fudan University, Shanghai 200031, China \\ Contributions: (I) Conception and design: X Xiao, S Zhang, H Chen; (II) Administrative support: J Yang; (III) Provision of study materials or \\ patients: X Xiao, S Zhang, J Yang, H Chen; (IV) Collection and assembly of data: All authors; (V) Data analysis and interpretation: All authors; (VI) \\ Manuscript writing: All authors; (VII) Final approval of manuscript: All authors. \\ \#These authors contributed equally to this work. \\ Correspondence to: Hao Chen, MD, PhD. Department of Thoracic Surgery, Xuhui Branch of Zhongshan Hospital, Fudan University, 966 Middle \\ Huaihai Road, Shanghai 200031, China. Email: h.chen@fudan.edu.cn.
}

Background: Nonoperative treatment is currently the standard therapy for rib fractures. However, there is a trend towards surgical fixation from conservative management over the last decade. While surgical fixation of rib fractures has shown promising results, its impact on the clinical results remains unclear based on the current literature. As such, the present study aims to compare the short-term outcomes of multiple rib fracture patients treated by surgical fixation with traditional conservative management.

Methods: Data for patients with multiple (three or more) rib fractures admitted to our department between January 2012 and January 2019 were retrospectively collected and analyzed. Propensity score matched patients were compared between those treated with surgical rib fixation and those of nonoperatively treated. Primary outcomes were hospital length of stay for multiple rib fracture patients, and intensive care unit (ICU) length of stay for flail chest patients. Secondary outcomes included in hospital mortality, ICU usage rate, duration of ventilator support, ventilator usage rate, and pneumonia.

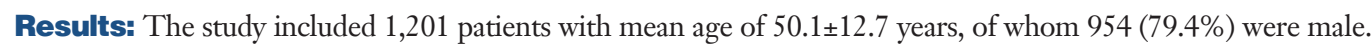
The average number of rib fractures was $6.3 \pm 2.4$, with a mean injury severity score of $20.5 \pm 7.3$. Among them, $563(46.9 \%)$ patients had surgical rib fixation and $638(53.1 \%)$ patients received nonoperative treatment. There were 191 patients with a flail chest, 133 (69.6\%) had operative rib fixation and 58 (30.4\%) were nonoperatively treated. After propensity score match, the hospital length of stay was not significantly differed between surgery and conservative management in multiple rib fracture patients $(10.7 \pm 3.4$ ss. $10.2 \pm 3.8$ days, $\mathrm{P}=0.067)$, nor were the secondary outcomes, in terms of in hospital mortality ( $0.9 \%$ vs. $1.1 \%, \mathrm{P}=0.704)$, ICU usage rate $(12.3 \%$ vs. $12.9 \%$, $\mathrm{P}=0.820)$, duration of ventilator support $(100.1 \pm 13.9$ vs. $99.8 \pm 20.7$ hours, $\mathrm{P}=0.822)$, ventilator usage rate $(12.0 \%$ vs. $12.9 \%, \mathrm{P}=0.732)$, and pneumonia $(24.3 \%$ vs. $24.9 \%, \mathrm{P}=0.861)$. For patients with a flail chest, shorter ICU length of stay was found to be associated with surgical rib fixation comparing with nonoperative treatment $(5.5 \pm 1.9$ vs. $6.7 \pm 2.1$ days, $\mathrm{P}=0.011)$. No secondary outcomes such as in hospital mortality $(4.4 \%$ vs. $4.4 \%, \mathrm{P}=1.000)$, ICU usage rate $(20.0 \%$ vs. $22.2 \%, \mathrm{P}=0.796)$, duration of ventilator support $(113.1 \pm 20.4$ vs. $131.2 \pm 19.5$ hours, $\mathrm{P}=0.535)$, ventilator usage rate $(20.0 \%$ vs. $20.0 \%, \mathrm{P}=1.000)$, pneumonia $(28.9 \%$ vs. $31.1 \%, \mathrm{P}=0.818)$ were significant different between the operative and nonoperative groups.

Conclusions: Surgical rib fixation results in a shorter ICU length of stay in patients with a flail chest, and a comparable outcome for patients with multiple rib fractures when compared with nonoperative treatment.

Keywords: Thoracic trauma; rib fractures; surgical rib fixation; flail chest

Submitted Oct 02, 2019. Accepted for publication Dec 11, 2019.

doi: 10.21037/atm.2020.01.39

View this article at: http://dx.doi.org/10.21037/atm.2020.01.39 


\section{Introduction}

Thoracic trauma is responsible for approximately $25 \%$ of trauma associated mortalities $(1,2)$. Rib fractures are the most common type of thoracic injuries following blunt trauma (3). It was reported that over 350,000 patients sustain rib injuries annually in the United States (4). Although there is currently no national database available in China, the number is estimated to reach the range of 1.5 to 2 million yearly, considering China's population and candidly its current noisy traffic and industrial security status $(5,6)$.

Rib fractures result in pain, paradoxical movement of chest wall, impaired ventilation, and respiratory failure. The current standard therapy for rib fractures mainly includes pain control, ventilation and pulmonary hygiene. Despite efforts have being spent, rib fractures, either alone or in combination with other concomitant injuries, are still associated with significantly morbidity and mortality. Clinical outcomes remain unsatisfactory and have not changed substantially over the last two decades (4).

With the advent of commercially available rib-specific fixation systems, surgical management of severe rib fractures, especially for flail chest, has recently gained increasing recognition (3). Multiple authors reported that surgical fixation of rib fractures is a safe and practical technique to restore chest wall stability and improve pulmonary function. However, there remains paucity of literature to describe its clinical value, in particular when compared with current nonoperative regimens. The aim of the study is therefore to compare the short-term outcomes of multiple rib fracture patients treated by surgical fixation with traditional nonoperative management.

\section{Methods}

\section{Study population}

Clinical records of patients with thoracic trauma who were admitted and treated in the department of thoracic surgery of a 1,030 bed tertiary hospital serving over 1.5 million residents between January 2012 and January 2019 were retrospectively retrieved and reviewed. All adult patients (18 years or older) with multiple rib fractures (defined as three or more rib fractures) were included in the present study. Patients who were below 18 years age, with fewer than three fractured ribs, with no chest computed tomography (CT) scans available, treated only in an outpatient setting or transferred to another institution were excluded. The study protocol was approved by our institutional review board. Patients consent was waived.

\section{Treatment}

Patients were admitted to general ward or intensive care unit (ICU) depending on their clinical situation. After admission, patients were evaluated by systemic physical examination, chest CT, electrocardiography and blood tests. Tube thoracostomy was performed in patients with pneumothorax or hemopneumothorax per surgeon's discretion to remove air, fluid, or blood. General inhospital surveillance included monitoring of respiration, heart rate, arterial blood pressure, central venous pressure, urine output, thoracic drainage, arterial gas analyses, etc. Patients were also evaluated in terms of coexisting pathologies and followed up closely by concerned specialists. Nonoperative treatment essentially consisted of oxygen inhalation, mechanical ventilation if indicated, pain control (includes oral or intravenous nonsteroidal anti-inflammatory analgesics, and loco-regional anesthesia such as intercostal nerve blocks and thoracic epidural catheters), bronchodilators and mucolytics, and antibiotics if indicated.

Indications for surgery were flail chest (defined as three or more consecutive ribs have multiple fractures within each rib, with clinical signs of paradoxical chest wall movement), multiple, severe displaced fractures, requiring video-assisted thoracoscopic surgery or thoracotomy, or failed early, optimal conservative management. Surgeries were usually performed within 72 hours of injury provided that the confounding factors are under controlled and the patient's general condition permit operation. For some patients with hemodynamics instability, or other higher priority injuries, rib fixations were performed within 7 days from injury. Preoperative planning was done using chest CT. All patients received antimicrobial prophylaxis (2.25 $\mathrm{g}$ of cefuroxime intravenously) within $30 \mathrm{~min}$ of incision.

Surgical rib fixations were performed by senior attending thoracic surgeons. In brief, patient was placed in a position to allow optimal access to rib fractures (usually lateral or posterior decubitus, and supine or prone in some cases). The incision placement was based upon the fracture pattern, following skin incision, a subcutaneous flap development provided access to the fracture, with muscle sparring technique applied whenever possible. The anterior soft tissue envelope of the rib approximately $2 \mathrm{~cm}$ on both sides of the fracture was removed, with the periosteum remained intact 
whenever possible, and an anatomical reduction was then performed using a titanium outer unicortical plate system (Seemine rib connector, Lanzhou, China). Closure included interrupted absorbable sutures to close muscular window and a closed suction drain in subcutaneous space. The selection of ribs for repair was at the discretion of the surgeon, with the aiming of restore chest wall stability and maintain effective oxygenation. Patients were encouraged to ambulate and mobilize as soon as possible after surgery. Post-operative care was similar to that of nonoperative patients, such as pain control and aggressive pulmonary toilet regimen.

The same group of thoracic surgeons managed both the operative and the nonoperative group. All patients had follow-up visits 2 and 4 weeks after discharge, with chest CT done upon the visits.

\section{Clinical outcomes comparison}

The clinical records including medical images of all enrolled patients upon admission were retrieved from our clinical information center electronically, and reviewed in terms of age, gender, etiology, injury type, rib fracture patterns such as number of rib fractures, unilateral/bilateral rib fractures, locations of rib fractures (upper/middle/lower third of the thorax), concomitant injuries, injury severity score (ISS), and treatment received. In addition, surgery-related characteristics were collected and reviewed in terms of time until surgery in days, duration of surgery in minutes, the number of surgically fixed rib fractures, and surgical-related complications such as surgical site bleeding and infection after rib fixation.

To describe the clinical values of surgical rib fixation in comparison with traditional nonoperative therapy, hospital length of stay, and ICU length of stay were served as the primary outcome measures for multiple rib fracture patients and flail chest patients, respectively. For both patient groups, secondary outcomes parameters included in hospital mortality, ICU usage rate, duration of ventilation support, ventilator usage rate, and pneumonia (defined by fever, hypopnea, coughing, or expectoration, and prescribed antibiotic focused on pulmonary organisms).

\section{Statistical analysis}

Statistical analyses were performed separately for patients with multiple rib fractures and patients with flail chest. To minimize potential confounding bias, propensity-matched analysis was applied to identify a group of nonoperative patients whose injuries were similar to that of the operative patients. Propensity scores were computed using a logistic prediction of surgical rib fixation as the dependent variable with the following covariates: age, gender, injury mechanism, number of rib fractures, unilateral/bilateral rib fractures, locations of rib fractures, concomitant injuries, and ISS scores. The 1:1 match was achieved using the nearest neighbor matching algorithm. Patients were considered being matched if the absolute difference in their propensity scores was less than 0.05 .

The categorical variables were presented as frequencies and percentages, and compared using the chi-squared test. Continuous variables were presented as mean \pm standard deviation (SD), tested for normality using Shapiro-Wilk normality test, and compared using the student's unpaired $t$-test (for parametric distribution) or the Mann-Whitney U-test (for nonparametric distribution), as appropriate. Two tailed $\mathrm{P}$ value of less than 0.05 was considered statistically significant. Data analysis was carried out using the Statistical Package for Social Sciences version 20 for Mac (SPSS Inc., Chicago, IL, USA).

\section{Results}

\section{Patient characteristics}

During the study period, a total of 1,201 patients were admitted with chest injury resulting in multiple rib fractures, of them 191 patients were diagnosed with a flail chest. There were 954 male and 247 female patients whose ages ranged from 18 years to 103 years with a mean age of 50.1 years. The average number of rib fractures was $6.3 \pm 2.4$, with a mean injury severity score of $20.5 \pm 7.3$. The major injury mechanism was high energy trauma (60.1\%), which includes road traffic accident, high impact falls and industrial accident. Before propensity matching, the baseline characteristics of surgically treated multiple rib fractures patients had more males and sustained a higher rate of co-existing hemothorax than those of the nonoperatively treated patients $(84.2 \%$ vs. $79.1 \%, \mathrm{P}=0.042$, and $27.2 \%$ vs. $22.1 \%, \mathrm{P}=0.001$, respectively). The surgically treated flail chest patients were consisted of more males and had a greater number of rib fractures than those of nonoperative patients $(75.2 \%$ vs. $56.9 \%, \mathrm{P}=0.011$, and $8.0 \pm 2.7$ vs. $7.1 \pm 2.1$, $\mathrm{P}=0.014$, respectively) (Table 1 ).

Of the 1,010 patients with multiple rib fractures, 430 patients had surgical rib fixation and 580 patients received nonoperative treatment. For 191 patients with 
Table 1 Characteristics of patients with multiple rib fractures or flail chest separated by treatment

\begin{tabular}{|c|c|c|c|c|c|c|c|}
\hline Characteristics & Overall $(n=1,201)$ & \multicolumn{3}{|c|}{ Multiple rib fractures } & \multicolumn{3}{|c|}{ Flail chest } \\
\hline Gender, n (\%) & & & & 0.042 & & & 0.011 \\
\hline Male & $954(79.4)$ & $362(84.2)$ & $459(79.1)$ & & $100(75.2)$ & $33(56.9)$ & \\
\hline Age (year), mean \pm SD & $50.1 \pm 12.7$ & $50.2 \pm 10.4$ & $49.1 \pm 9.3$ & 0.083 & $52.1 \pm 9.7$ & $49.2 \pm 9.1$ & 0.054 \\
\hline Injury mechanism, n (\%) & & & & 0.592 & & & 0.761 \\
\hline High energy trauma & $722(60.1)$ & $246(57.2)$ & $322(55.5)$ & & $108(81.2)$ & $46(79.3)$ & \\
\hline Other & 479 (39.9) & $184(42.8)$ & $258(44.5)$ & & $25(18.8)$ & $12(20.7)$ & \\
\hline Bilateral & $425(35.4)$ & $162(37.7)$ & $205(35.3)$ & & $39(29.3)$ & $19(32.8)$ & \\
\hline Rib fracture locations, n (\%) & & & & 0.101 & & & 0.309 \\
\hline Upper & $192(15.9)$ & $74(17.2)$ & $88(15.2)$ & & $21(15.8)$ & $9(15.5)$ & \\
\hline Middle & $845(70.4)$ & $303(70.5)$ & $393(67.8)$ & & $106(79.7)$ & $43(74.1)$ & \\
\hline Lower & $164(13.7)$ & $53(12.3)$ & $99(17.1)$ & & $6(4.5)$ & $6(10.3)$ & \\
\hline \multicolumn{8}{|l|}{ Concomitant injuries, n (\%) } \\
\hline Pneumothorax & $667(55.5)$ & $230(53.5)$ & $320(55.2)$ & 0.595 & $81(60.9)$ & $36(60.1)$ & 0.879 \\
\hline
\end{tabular}

Categorical data are presented as number and percent; continuous data are presented as mean \pm SD. SD, standard deviation.

a flail chest, 133 had operative rib fixation and 58 were nonoperatively treated. Of the 563 operatively treated patients, 107 underwent surgical rib fixation within $24 \mathrm{~h}$, 378 between 24 and $72 \mathrm{~h}$ and all within 7 days after hospital admission. The average duration of surgery was $153 \pm 16 \mathrm{~min}$. A median number of 4 ribs was fixated. No intra-operative complications were occurred. Surgical procedure related complications were not substantial: bleeding necessitated surgical intervention occurred in 5 patients with multiple rib fractures and 2 patients with flail chest, all within $24 \mathrm{~h}$ after rib fixation. Wound infections requiring drainage or debridement were recorded in 31 and 12 patients of multiple rib fractures and flail chest, respectively, between 3 to 7 days after surgeries (Table 2). All patients were discharged home and were uneventful with chest CT done upon the visits at 2 - and 4 -week post discharge.

\section{Clinical outcome comparison-before propensity score match}

\section{Primary outcomes}

The primary outcome for multiple rib fractures, the hospital length of stay, was not significantly differed between surgically and conservatively managed patients with multiple rib fractures $(10.3 \pm 3.6$ vs. $10.1 \pm 4.5$ days, $\mathrm{P}=0.433)$. The ICU length of stay, as the primary outcome for flail chest, was significantly differed between surgery and conservative management in patients with a flail chest (5.7 \pm 2.1 vs. $6.6 \pm 2.4$ days, $\mathrm{P}=0.015$ ). 
Table 2 Surgical characteristics and surgery related complications of patients with multiple rib fractures or flail chest

\begin{tabular}{|c|c|c|c|}
\hline Characteristics & Overall $(n=563)$ & Multiple rib fracture $(n=430)$ & Flail chest $(n=133)$ \\
\hline$<24$ hours & $107(19.0)$ & $66(15.3)$ & $41(30.8)$ \\
\hline $24-72$ hours & $378(67.1)$ & $311(72.3)$ & $67(50.4)$ \\
\hline 72 hours to 7 days & $78(13.9)$ & $53(12.3)$ & $25(18.8)$ \\
\hline Number of surgically fixed rib fractures (mean \pm SD) & $4.0 \pm 1.3$ & $3.8 \pm 1.4$ & $4.7 \pm 1.6$ \\
\hline \multicolumn{4}{|l|}{ Surgical complication, n (\%) } \\
\hline Bleeding & $7(1.2)$ & $5(1.2)$ & $2(1.5)$ \\
\hline Wound infection & $43(7.6)$ & $31(7.2)$ & $12(9.0)$ \\
\hline
\end{tabular}

Categorical data are presented as number and percent; continuous data are presented as mean \pm SD. SD, standard deviation.

Table 3 Clinical outcome for patients with multiple rib fractures or flail chest separated by treatment, before propensity score match

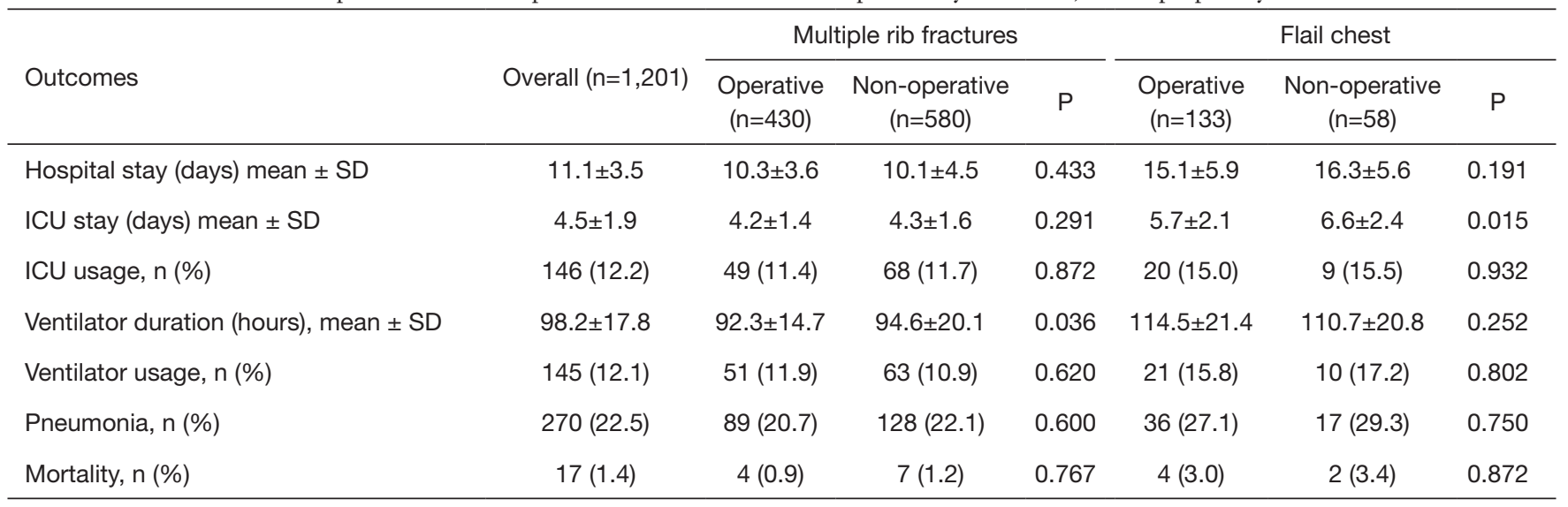

Categorical data are presented as number and percent; continuous data are presented as mean $\pm \mathrm{SD}$. SD, standard deviation.

\section{Secondary outcomes}

The secondary outcomes of multiple rib fractures, in terms of ICU length of stay $(4.2 \pm 1.4$ vs. $4.3 \pm 1.6$ days, $\mathrm{P}=0.291)$, in hospital mortality $(0.9 \%$ vs. $1.2 \%, \mathrm{P}=0.767)$, ICU usage rate $(11.4 \%$ vs. $11.7 \%, \mathrm{P}=0.872)$, ventilator usage rate (11.9\% vs. $10.9 \%, \mathrm{P}=0.620)$, and pneumonia $(20.7 \%$ vs. $22.1 \%, \mathrm{P}=0.600)$ were similar between 2 groups, except that the operative patients had a shorter duration of ventilator support than nonoperative patients $(92.3 \pm 14.7 \mathrm{vs}$. 94.6 \pm 20.1 hours, $\mathrm{P}=0.036$ ). The secondary outcomes of flail chest, including in hospital mortality $(3.0 \%$ vs. 3.4\%, $\mathrm{P}=0.872)$, ICU usage rate $(15.0 \%$ vs. $15.5 \%, \mathrm{P}=0.932)$, duration of ventilator support $(114.5 \pm 21.4$ vs. $110.7 \pm 20.8$ hours, $\mathrm{P}=0.252)$, ventilator usage rate $(15.8 \%$ vs. $17.2 \%$, $\mathrm{P}=0.802)$, and pneumonia $(27.1 \%$ vs. $29.3 \%, \mathrm{P}=0.750)$, were not remarkably differed between operative and nonoperative patients, nor was the hospital length of stay (15.1 \pm 5.9 vs. $16.3 \pm 5.6$ days, $\mathrm{P}=0.191)$ (Table 3).

\section{Clinical outcome comparison-after propensity score match}

Of the surgically treated patients, 350 patients with multiple rib fractures and 45 patients with a flail chest were successfully matched to a corresponding nonoperatively treated patient group, respectively. No significant differences were found between them regarding the baseline characteristics (Table 4).

\section{Primary outcomes}

The primary outcome for multiple rib fractures, the hospital length of stay, was not significantly differed 
Table 4 Characteristics of propensity matched patients with multiple rib fractures or flail chest separated by treatments

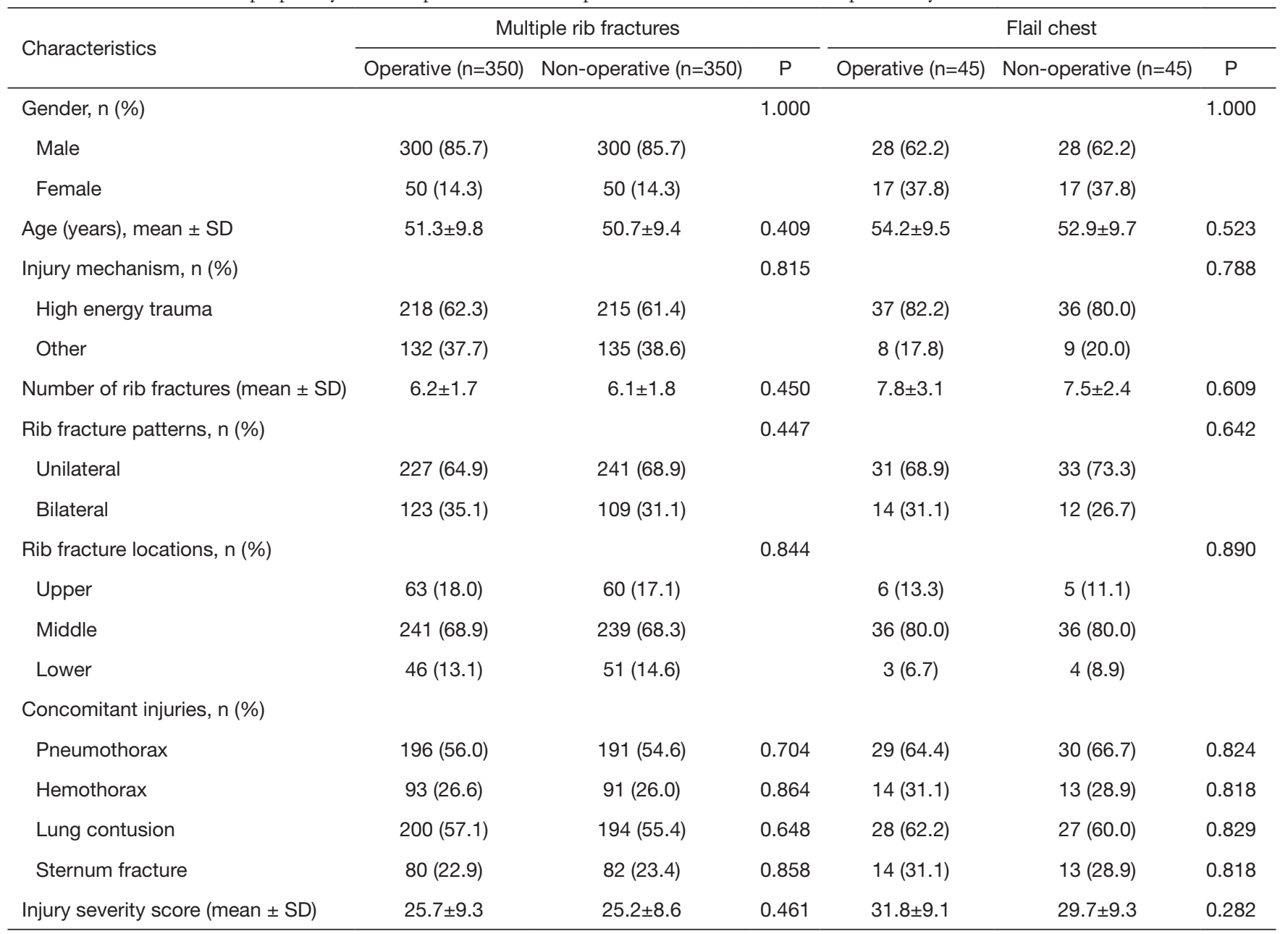

Categorical data are presented as number and percent; continuous data are presented as mean \pm SD. SD, standard deviation.

between surgically and conservatively managed multiple rib fractures patients $(10.7 \pm 3.4$ vs. $10.2 \pm 3.8$ days, $\mathrm{P}=0.067)$. The ICU length of stay, as the primary outcome for patients with a flail chest, was significantly differed between surgery and conservative management flail chest patients $(5.5 \pm 1.9$ vs. $6.7 \pm 2.1$ days, $\mathrm{P}=0.011$ ).

\section{Secondary outcomes}

No significant differences were found between the operative and nonoperative multiple rib fracture patients for secondary outcome parameters, including in hospital mortality $(0.9 \%$ vs. $1.1 \%, \mathrm{P}=0.704)$, ICU usage rate (12.3\% vs. $12.9 \%, \mathrm{P}=0.820$ ), duration of ventilator support $(100.1 \pm 13.9$ vs. $99.8 \pm 20.7$ hours, $\mathrm{P}=0.822)$, ventilator usage rate $(12.0 \%$ vs. $12.9 \%, \mathrm{P}=0.732)$, and pneumonia $(24.3 \%$ vs. $24.9 \%, \mathrm{P}=0.861)$, nor was the ICU length of stay $(4.3 \pm 1.5$ vs. $4.2 \pm 1.8$ day, $\mathrm{P}=0.425)$.

No significant differences were found between operative and nonoperative patients with a flail chest in terms of in hospital mortality ( $4.4 \%$ vs. 4.4\%, $\mathrm{P}=1.000$ ), ICU usage rate (20.0\% vs. $22.2 \%, \mathrm{P}=0.796$ ), duration of ventilator support $(113.1 \pm 20.4$ vs. $131.2 \pm 19.5$ hours, $\mathrm{P}=0.535)$, ventilator usage rate $(20.0 \%$ vs. $20.0 \%, \mathrm{P}=1.000)$, pneumonia $(28.9 \%$ vs. $31.1 \%, \mathrm{P}=0.818)$, and the hospital length of stay $(16.7 \pm 6.1$ vs. $16.8 \pm 5.9$ days, $\mathrm{P}=0.937$ ) (Table 5).

\section{Discussion}

The present study compared the clinical outcomes of surgical rib fixation with conventional conservative management for both multiple rib fractures and flail chest patients. The crude data showed that rib fixation could reduce ventilator 
Table 5 Clinical outcome for patients with multiple rib fractures or flail chest separated by treatment, after propensity score match

\begin{tabular}{|c|c|c|c|c|c|c|}
\hline Outcomes & \multicolumn{3}{|c|}{ Multiple rib fractures } & \multicolumn{3}{|c|}{ Flail chest } \\
\hline ICU stay (days), mean \pm SD & $4.3 \pm 1.5$ & $4.2 \pm 1.8$ & 0.425 & $5.5 \pm 1.9$ & $6.7 \pm 2.1$ & 0.011 \\
\hline Ventilator usage, n (\%) & $42(12.0)$ & $45(12.9)$ & 0.732 & $9(20.0)$ & $9(20.0)$ & 1.000 \\
\hline Pneumonia, n (\%) & $85(24.3)$ & 87 (24.9) & 0.861 & $13(28.9)$ & $14(31.1)$ & 0.818 \\
\hline Mortality, n (\%) & $3(0.9)$ & $4(1.1)$ & 0.704 & $2(4.4)$ & $2(4.4)$ & 1.000 \\
\hline
\end{tabular}

Categorical data are presented as number and percent; continuous data are presented as mean \pm SD. SD, standard deviation.

support time in multiple rib fractures patients, and gained a significant shorter ICU length of stay in patients with a flail chest. After adjusting for potential confounding variables, rib fixation was found not associated with differences in terms of hospital length of stay or other secondary outcome measurements in patients with multiple rib fractures. However, rib fixation was effective to reduce ICU length of stay for flail chest patients, although no statistical difference was found for other secondary outcome parameters.

Rib fractures are the most common type of thoracic traumas, result in pain, paradoxical respiration, compromised ventilation, and account for significant morbidity and mortality. Despite tremendous efforts spent over years, its clinical outcome remains unsatisfactory, which highlights and necessitates continually treatment modalities innovations. With the advances of rib fixation systems, surgical management for severe rib fractures has recently gained increasing acceptances $(3,7)$. It is speculated that surgical rib fixation could provide a faster recovery by restoring stability of the thorax wall and consequently maintaining effective air exchange. In addition, surgical rib fixation is supposed to be helpful for an optimal pain control, so that minimizes chances of pulmonary and immobility related complications. Although many studies have reported its potentials, its clinical value, in particular its safety and efficacy, as compared to current conservative regimens, is still controversial and under critical evaluation (7-10).

To our knowledge, this is the one of the largest studies to compare surgical rib fixation with conventional management in a statistically matched cohort of patients. Rib fracture patients enrolled in present study were predominantly male, had similar age, fracture distribution, co-existing injuries and comparable injury severity scores, except for that the operative patients (either multiple rib fractures or flail chest) had more males, the operative flail chest patients had higher number of rib fractures, and the operative multiple rib fracture patients had more hemothorax, when compared to their controls, respectively. After propensity score match, all these baseline characteristics were comparable between operative and nonoperative groups. Despite of marginal benefits, surgery was found not associated with significant clinical outcome improvement in multiple rib fractures patients. On the other hand, in patients with a flail chest, surgical rib fixation did remarkably reduce the ICU length of stay by approximately 1.2 days, as compared to nonoperative group.

There is a bunch of evidence supports the advantages of surgical rib fixation over traditional conservative regimes, including several randomized controlled trials, whose authors reported the beneficial effects of surgical rib fixation, such as significant shorter duration of ventilation support, lower rate of pneumonia, and shorter ICU and hospital length of stay (11-15). However, these studies enrolled relatively much smaller number of patients, and might have much stricter inclusion criteria than that of current study. Instead, the present study investigated all patients with multiple rib fractures or a flail chest, including those who have companion injuries to other organ systems, such as extremity fracture, abdominal injury, and craniocerebral trauma, etc., that might be more representative in real world clinic scenario, although inevitably compromised homogeneity of the study subjects as a tradeoff.

This study should be viewed and interpreted in the light of its limitations. The retrospective and observational 
nature of the study inherent to all known associated shortcomings, and might have compromised the affirmative conclusion, as such future large scale randomized controlled studies are obviously warranted (7). Besides, the variation of management strategies during the study period might have potentially biased the patient selection and decision making, and made it difficult for comparisons with other investigators, considering there is still no national practice guideline nor consensus for the management of this patient group in China. Furthermore, the current outcome parameters might not be able to detect relatively small but clinically meaningful differences. Pain control, as an important outcome measurement, was unable to be assessed due to the retrospective study design. Lastly, the short follow-up period might not allow adequate evaluation of regime associated complications such as symptomatic fixation material or chronic bone nonunion. Despite these limitations, the present study, however, adds valuable evidence on the clinical value of surgical rib fixation, and emphasizes the need for further randomized controlled studies.

\section{Conclusions}

This large sample, propensity score matched study shows that surgical rib fixation is safe and improves short-term outcome in terms of ICU length of stay in patients with a flail chest, as compared to traditional conservative therapy. For patients with multiple rib fractures, surgical rib fixation results in a comparable outcome in comparison with nonoperative treatment. Future randomized controlled studies are needed to define its clinical significance.

\section{Acknowledgments}

This study was undertaken at the Institute of Clinical Science, Zhongshan Hospital, Fudan University.

Funding: This work was supported by research grants from the Science and Technology Commission of Shanghai Municipality (18ZR1407200), Shanghai Municipal Health Commission (201840351), National Natural Science Foundation of China (81470282 and 81170473), Science and Technology Commission of Qingpu District (QKY2017-03), and Qingpu District Health Commission (W2017-21).

\section{Footnote}

Conflicts of Interest: The authors have no conflicts of interest to declare.

Ethical Statement: The authors are accountable for all aspects of the work in ensuring that questions related to the accuracy or integrity of any part of the work are appropriately investigated and resolved. The current study was approved by the Institution Review Board of Zhongshan Hospital Qingpu Branch, Fudan University (ref \# 2018-11). All patient's personal data have been secured and the patient consents were waived.

Open Access Statement: This is an Open Access article distributed in accordance with the Creative Commons Attribution-NonCommercial-NoDerivs 4.0 International License (CC BY-NC-ND 4.0), which permits the noncommercial replication and distribution of the article with the strict proviso that no changes or edits are made and the original work is properly cited (including links to both the formal publication through the relevant DOI and the license). See: https://creativecommons.org/licenses/by-nc-nd/4.0/.

\section{References}

1. Ziegler DW, Agarwal NN. The morbidity and mortality of rib fractures. J Trauma 1994;37:975-9.

2. Sirmali $M$, Turut $H$, Topcu $S$, et al. A comprehensive analysis of traumatic rib fractures: morbidity, mortality and management. Eur J Cardiothorac Surg 2003;24:133-8.

3. Pieracci FM, Majercik S, Ali-Osman F, et al. Consensus statement: Surgical stabilization of rib fractures rib fracture colloquium clinical practice guidelines. Injury 2017;48:307-21.

4. Dehghan N, de Mestral C, McKee MD, et al. Flail chest injuries: a review of outcomes and treatment practices from the National Trauma Data Bank. J Trauma Acute Care Surg 2014;76:462-8.

5. Zhang S, Tang M, Ma J, et al. Thoracic trauma: a descriptive review of 4168 consecutive cases in East China. Medicine (Baltimore) 2019;98:e14993.

6. He W, Yang Y, Wu W, et al. Chest wall stabilization (CWS) in China: current situation and prospect. J Thorac Dis 2019;11:S1044-8.

7. Pieracci FM, Leasia K, Whitbeck S. Barriers to conducting a multi-center randomized controlled trial of surgical stabilization of rib fractures (and how to overcome them). J Thorac Dis 2019;11:S1049-60.

8. Beks RB, Peek J, de Jong MB, et al. Fixation of flail chest or multiple rib fractures: current evidence and how to 
proceed. A systematic review and meta-analysis. Eur J Trauma Emerg Surg 2019;45:631-44.

9. Witt CE, Bulger EM. Comprehensive approach to the management of the patient with multiple rib fractures: a review and introduction of a bundled rib fracture management protocol. Trauma Surg Acute Care Open 2017;2:e000064.

10. Beks RB, Reetz D, de Jong MB, et al. Rib fixation versus non-operative treatment for flail chest and multiple rib fractures after blunt thoracic trauma: a multicenter cohort study. Eur J Trauma Emerg Surg 2019;45:655-63.

11. Liu T, Liu P, Chen J, et al. A Randomized Controlled Trial of Surgical Rib Fixation in Polytrauma Patients With Flail Chest. J Surg Res 2019;242:223-30.

12. Marasco SF, Davies AR, Cooper J, et al. Prospective

Cite this article as: Xiao X, Zhang S, Yang J, Wang J, Zhang Z, Chen H. Surgical fixation of rib fractures decreases intensive care length of stay in flail chest patients. Ann Transl Med 2020;8(5):216. doi: 10.21037/atm.2020.01.39 randomized controlled trial of operative rib fixation in traumatic flail chest. J Am Coll Surg 2013;216:924-32.

13. Tanaka H, Yukioka T, Yamaguti Y, et al. Surgical stabilization of internal pneumatic stabilization? A prospective randomized study of management of severe flail chest patients. J Trauma 2002;52:727-32; discussion 732.

14. Khandelwal G, Mathur RK, Shukla S, et al. A prospective single center study to assess the impact of surgical stabilization in patients with rib fracture. Int J Surg 2011;9:478-81.

15. Granetzny A, Abd El-Aal M, Emam E, et al. Surgical versus conservative treatment of flail chest. Evaluation of the pulmonary status. Interact Cardiovasc Thorac Surg 2005;4:583-7. 